\title{
Current-voltage characteristics of carbon nanotubes with substitutional nitrogen
}

Kaun, C.C.; Larade, B.; Mehrez, H.; Taylor, Jeremy Philip; Guo, H.

Published in:

Physical Review B Condensed Matter

Link to article, DOI:

10.1103/PhysRevB.65.205416

Publication date:

2002

Document Version

Publisher's PDF, also known as Version of record

Link back to DTU Orbit

Citation (APA):

Kaun, C. C., Larade, B., Mehrez, H., Taylor, J. P., \& Guo, H. (2002). Current-voltage characteristics of carbon nanotubes with substitutional nitrogen. Physical Review B Condensed Matter, 65(20), 205416.

https://doi.org/10.1103/PhysRevB.65.205416

\section{General rights}

Copyright and moral rights for the publications made accessible in the public portal are retained by the authors and/or other copyright owners and it is a condition of accessing publications that users recognise and abide by the legal requirements associated with these rights.

- Users may download and print one copy of any publication from the public portal for the purpose of private study or research.

- You may not further distribute the material or use it for any profit-making activity or commercial gain

- You may freely distribute the URL identifying the publication in the public portal 


\title{
Current-voltage characteristics of carbon nanotubes with substitutional nitrogen
}

\author{
Chao-Cheng Kaun, ${ }^{1}$ Brian Larade, ${ }^{1}$ Hatem Mehrez, ${ }^{1}$ Jeremy Taylor, ${ }^{2,1}$ and Hong Guo \\ ${ }^{1}$ Center for the Physics of Materials and Department of Physics, McGill University, Montreal, PQ H3A 2T8, Canada \\ ${ }^{2}$ Mikroelektronik Centret (MIC), Technical University of Denmark, East DK-2800 Kohgens Lyngby, Denmark
}

(Received 30 November 2001; revised manuscript received 08 March 2002; published 14 May 2002)

\begin{abstract}
We report ab initio analysis of current-voltage $(I-V)$ characteristics of carbon nanotubes with nitrogen substitution doping. For zigzag semiconducting tubes, doping with a single $\mathrm{N}$ impurity increases current flow and, for small radii tubes, narrows the current gap. Doping a $\mathrm{N}$ impurity per nanotube unit cell generates a metallic transport behavior. Nonlinear $I-V$ characteristics set in at high bias and a negative differential resistance region is observed for the doped tubes. These behaviors can be well understood from the alignment $/ \mathrm{mis}$ alignment of the current carrying bands in the nanotube leads due to the applied bias voltage. For a armchair metallic nanotube, a reduction of current is observed with substitutional doping due to elastic backscattering by the impurity.
\end{abstract}

Carbon nanotubes are either metals or semiconductors depending on their helicity and therefore have the capability of forming an all carbon nanotube-based molecular electronics system. ${ }^{1,2}$ As is the case for conventional semiconductor materials, doping a carbon nanotube with other atomic species provides a means of altering its electronic and transport properties. ${ }^{3-11}$ On the theoretical investigations of transport through doped nanotubes, almost all analyses so far have focused on evaluating equilibrium conductance of doped carbon nanotubes ${ }^{3,4,7-10}$ under various doping conditions and models. A more complete understanding of doped nanotubes also requires investigations of nonequilibrium transport properties such as the current-voltage $(I-V)$ characteristics, for which the studies were quite limited to date.,6

In this paper, we present an $a b$ initio self-consistent analysis of nonequilibrium transport through carbon nanotubes doped with atomic nitrogen in the form of substitution. The nonequilibrium condition is provided by a finite external bias voltage that drives a steady-state current flow. We note that existing investigations on transport through doped nanotubes were based on tight-binding or continuum models for dopants, ${ }^{5,6}$ here we report nonlinear $I-V$ characteristics of doped nanotubes from first principles. Nitrogen substitution has already attracted theoretical attention for its structural ${ }^{3}$ and equilibrium transport ${ }^{4,8}$ properties. It is interesting structurally because replacing a carbon by a nitrogen in an otherwise pristine nanotube does not cause substantial structural relaxation. ${ }^{3}$ It is also interesting transport wise as the substitution disorder provides elastic scattering centers that alter the steplike equilibrium conductance of pure nanotubes. ${ }^{4,10}$ Our calculation further suggests that nitrogen doping can have a profound nonlinear influence on the transport properties of semiconducting nanotubes. Our main results are the following.

(i) The current flowing through zigzag semiconducting nanotubes is increased by even a single substitutional nitrogen and, for small radius tubes, the current gap is narrowed.

(ii) For periodic doping of a $\mathrm{N}$ atom per unit cell of a semiconducting nanotube, transport becomes metallic and the $I-V$ curve shows a nonlinear behavior and a negative differential resistance (NDR). (iii) For an armchair metallic nanotube, doping with a single $\mathrm{N}$ impurity slightly reduces the current. Finally, all the nonequilibrium transport properties can be understood from the electronic structure of the doped carbon nanotube devices, and a simple physical picture emerges for these devices from the point of view of alignment or misalignment of the current carrying bands of the leads due to the applied bias voltage.

We consider carbon nanotubes doped with one or more nitrogen atoms, in the form of substitution of carbon by nitrogen. We neglect the very small structural relaxation due to the substitution. ${ }^{3}$ A bias voltage $V_{b}$ is applied across the nanotube device, which drives a steady-state current flowing along the tube axis. The entire system can be viewed as a device scattering region (where the atom substitution resides) connected to two nanotube leads. ${ }^{12,13}$ Exactly how nanotubes are doped will depend on various experimental conditions, and in this work we focus on the simplest model and the qualitative physics associated with nonequilibrium transport. Our investigation is based on a recently developed selfconsistent first-principles technique ${ }^{12}$ that combines the Keldysh nonequilibrium Green's function formalism ${ }^{14-16}$ (NEGF) with a real space self-consistent density-functional theory (DFT). ${ }^{13}$ For details of this method we refer interested readers to Ref. 12. The transmission coefficient $T\left(E, V_{b}\right)$, which is a function of energy $E$ and bias $V_{b}$, is calculated from Green's functions, ${ }^{15,12}$ and electric current is obtained as

$$
I=\frac{2 e}{h} \int_{\mu_{\min }}^{\mu_{\max }} d E\left(f_{l}-f_{r}\right) T\left(E, V_{b}\right),
$$

where $\mu_{\text {max } / \text { min }}$ is the maximum/minimum value of the electrochemical potential of the two leads and $f_{l, r}$ is the Fermi distribution function.

Figure 1 plots transport result for $(7,0)$ zigzag semiconducting nanotubes with and without doping of a single nitrogen. The equilibrium result, i.e., transmission coefficient at zero bias $T\left(E, V_{b}=0\right)$, is plotted versus scattering electron energy in Fig. 1(a) (Fermi energy is at $E_{F}=0$, dashed line). The changes of $T\left(E, V_{b}=0\right)$ for pure tubes (dotted line) cor- 

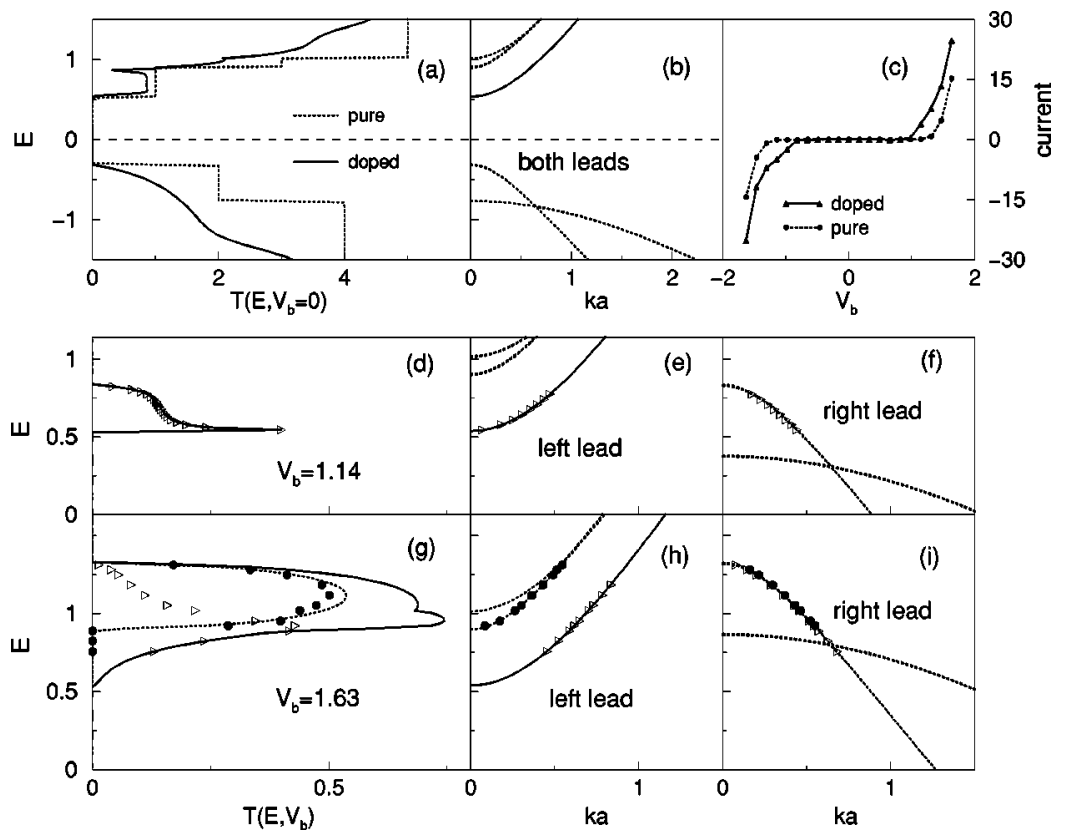

FIG. 1. For pure and single $\mathrm{N}$ doped $(7,0)$ nanotubes. (a) The equilibrium transmission coefficient versus energy. (b) The band structure of the lead versus energy where ka is the Bloch number: solid line is the hybridization band. The Fermi level is at $E_{F}=0$. (c) The $I-V$ curves. (d), (g) Evolution of the total transmission coefficient $T\left(E, V_{b}\right)$ under increasing bias voltage for pure tube (dotted line) and single $\mathrm{N}$-doped tube (solid line). The transmission coefficient for each conducting band for doped tube are also shown ( and $\triangleright$ ). (e), (h) Band structures of the left lead and (f), (i) for the right lead. The bands that contribute to conduction are indicated by symbols ( and $\triangleright$ ). The states in right lead are raised by bias voltage. The unit for energy is $\mathrm{eV}$, for bias voltage is $\mathrm{V}$, and for current is $\mu \mathrm{A}$. relate perfectly with the corresponding nanotube band structure $^{17}$ shown in Fig. 1(b): $T\left(E, V_{b}=0\right)$ jumps in steps of 2 because the bands are doubly degenerate; an exception being the "hybridization band" shown by the solid line [Fig. 1(b)], which comes from hybridization of graphitic states due to nanotube curvature. ${ }^{18} T\left(E, V_{b}=0\right)$ with the single nitrogen substitution [solid line, Fig. 1(a)], while following the same general trend, is somewhat reduced in value due to backscattering of electrons by the nitrogen impurity. ${ }^{10}$ Lammert et al. ${ }^{8}$ showed that doping a $\mathrm{N}$ impurity gives rise to impurity levels having energies within the energy gap of the semiconductor nanotube. These impurity levels do not align with bands of the pristine nanotube leads and therefore do not contribute to conduction in any appreciable way, i.e., the doping does not introduce a resonance transmission at an energy within the gap. More interesting is the result under nonequilibrium condition, shown in Figs. 1(d) and 1(g). Figure $1(\mathrm{~d})$ plots $T\left(E, V_{b}=1.14\right)$ versus $E$ in range $0<E<V_{b}$ [according to Eq. (1), only the bands inside this range can contribute to conduction]. For pure tube $T\left(E, V_{b}=1.14\right)=0$, but for doped tube $T\left(E, V_{b}=1.14\right) \neq 0$ and it appears different as compared to the equilibrium result. This behavior can be understood qualitatively from the point of view of band alignment of the nanotube leads. As $V_{b}$ (applied to the right lead) is increased from zero, the bands in the right lead [Fig. 1(f)] are shifted upwards by amount $V_{b}$ relative to those of the left lead ${ }^{5,6}$ [Fig. 1(e)]. Therefore, at $V_{b}=1.14 \mathrm{~V}$, the valence band of the right lead align (in energy) with the hybridization band of the left lead, and $T\left(E, V_{b}\right)$ is nonzero for doped tubes while is still zero for pure tubes. For a pure tube, $T\left(E, V_{b}\right)$ becomes nonzero only when the $\pi^{*}$ band of left lead (conduction band, not the hybridization band) is aligned with the $\pi$ band of right lead (valence band), because these bands have the same rotational symmetry. ${ }^{2,5,6,19}$ However, at $V_{b}=1.14 \mathrm{~V}$, these bands have not been aligned yet, therefore $T\left(E, V_{b}\right)$ remains zero at this voltage. For doped tubes, on the other hand, rotational symmetry is broken by the presence of impurity, and $T\left(E, V_{b}\right)$ becomes nonzero when any bands of left and right leads are aligned. Hence $T\left(E, V_{b}\right)$ starts to be nonzero for doped tubes at $V_{b}$ $\approx 0.81 \mathrm{~V}$ due to the starting of alignment of the left hybridization band to the right valence band. For the pure tube, conduction only starts when $V_{b} \approx 1.20 \mathrm{~V}$ at which the right valence band starts to align with the left conduction band. The significance of these results is that current becomes nonzero at $V_{b} \approx 0.81 \mathrm{~V}$ for doped tubes while remains zero until $V_{b} \approx 1.20 \mathrm{~V}$ for pure tubes, shown by the $I-V$ curves of Fig. 1(c). Therefore, even a single nitrogen doping has narrowed the current gap for $(7,0)$ tubes by a significant amount, and we can make the same conclusion for other zigzag tubes with radii less than that of the $(9,0)$ tube because all these small radii tubes have a hybridization band with $\Gamma$ point lower than that of their $\pi^{*}$ band. ${ }^{18}$ The conclusion also has implications on the magnitudes of current and transmission coefficients $T\left(E, V_{b}\right)$. For example, at $V_{b} \approx 1.63 \mathrm{~V}$ [Fig. $\left.1(\mathrm{~g})\right]$, both doped and pure $(7,0)$ tubes are conducting. However, for doped tubes both the left $\pi^{*}$ and hybridization bands contribute to transport, while only the $\pi^{*}$ contributes for the pure tubes. For doped tubes the individual transmission through the hybridization band (triangles) and the $\pi^{*}$ band (solid circles) are also shown for $V_{b}=1.63 \mathrm{~V}$ [Fig. $\left.1(\mathrm{~g})\right]$ : the contribution from the $\pi^{*}$ band is only slightly smaller than the total transmission through the pure tube (dotted line). This is why current and total transmission $T\left(E, V_{b}\right)$ take larger values for the doped tubes [Fig. 1(c)]. Finally, we have checked numerically that the individual channel transmission $\sim\left(v_{l} v_{r}\right)$, where $v_{l, r}=(1 / \hbar)(\partial E / \partial k)$ is the group velocity of left (right) lead (the slope of the band), ${ }^{20}$ a result that is consistent with the well known Fisher-Lee relationship. ${ }^{21}$

Next we consider $(7,0)$ tubes periodically doped with a nitrogen atom per nanotube unit cell, the results shown in Fig. 2. At equilibrium, i.e., $V_{b}=0$, there are three bands crossing $E_{F}$ so that $T\left(E_{f}, V_{b}=0\right)=3$ indicating a metallic 


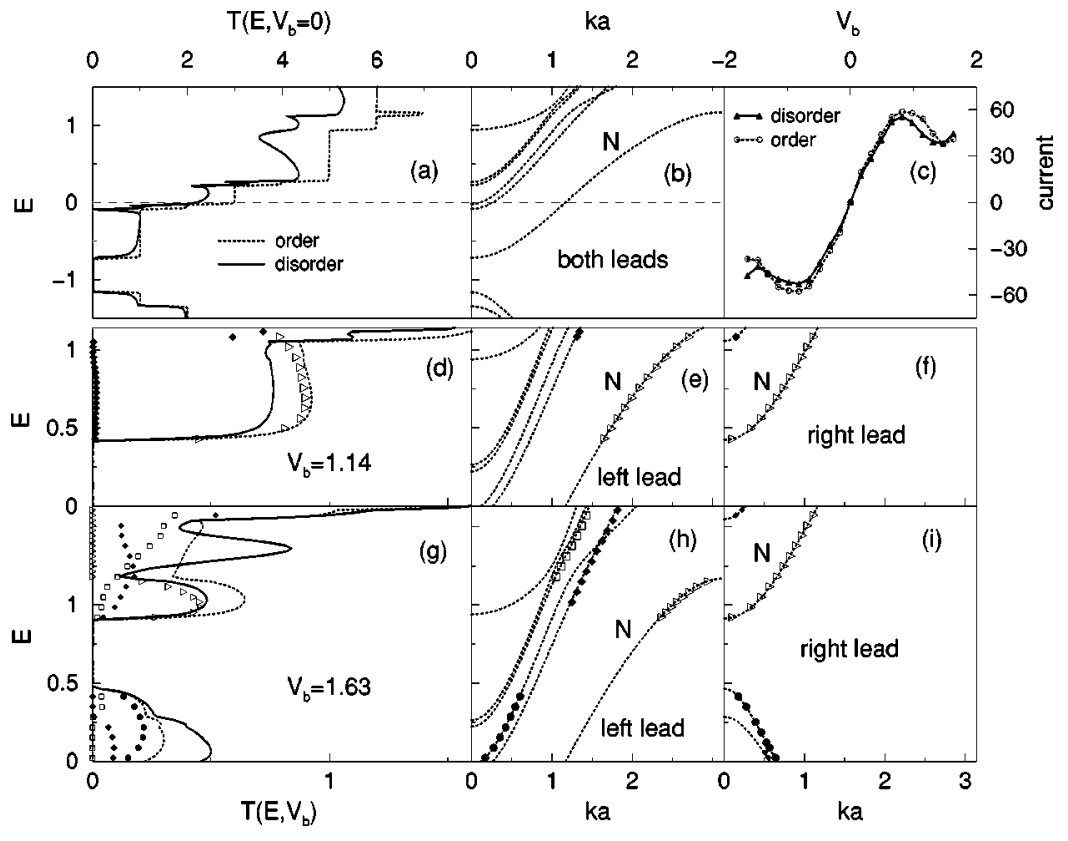

FIG. 2. For periodic N-doped $(7,0)$ nanotubes. In (a),(c),(d),(g), the solid line is for periodic doping but with one $\mathrm{N}$ atom located at a disordered position; the dotted line is for perfect periodic doping. (a) The equilibrium conductance; (b) the band structure of the lead; (c) the $I-V$ curve; (d), (g) the evolution of total transmission coefficient $T\left(E, V_{b}\right)$; and (e), (h), (f), (i) are for band structures of the left and right leads. Units are the same as in Fig. 1. behavior [Fig. 2(a)]. One of the bands that cuts $E_{F}$, labeled by $N$, is derived primarily from the nitrogen atomic state and this band is roughly half filled [see Fig. 2(b)]. Again, the steplike $T\left(E, V_{b}=0\right)$ curve [Fig. 2(a)] can be perfectly correlated with the band structure. Since each band is nondegenerate, $T\left(E, V_{b}=0\right)$ increases in steps of one unit. Figs. 2(d) and $2(\mathrm{~g})$ (dotted line) show nonequilibrium results of $T\left(E, V_{b}\right)$ for $V_{b}=1.14 \mathrm{~V}$ and $1.63 \mathrm{~V}$, respectively. As $V_{b}$ is increased from zero to $0.74 \mathrm{~V}$ (applied to the right lead), current $I$ increases from zero to maximum value because the band edge of the right $\mathrm{N}$ band is raised to align with the $E_{F}$ of the left lead, and the overlap between the $\mathrm{N}$ bands of left and right leads becomes maximum giving rise to the maximum current, ${ }^{5,6}$ shown in the $I-V$ curve of Fig. 2(c). When $V_{b}$ increases further, $I$ starts to decrease a little, because the slope of the left $\mathrm{N}$ band decreases at large energies [see Fig. 2(e)], NDR therefore appears. When $V_{b}$ increase to $1.16 \mathrm{~V}$, the N-band edge of left lead aligns with the $E_{F}$ of right lead, and the overlap of the $\mathrm{N}$ bands starts to decreases, as a result the current decreases further. Although there are some other bands (box, solid diamond, and solid circles, see Fig. 2(h) and 2(i) that can contribute to conduction, they cannot compensate for the loss of conduction due to the misalignment of the $\mathrm{N}$ bands. The overall result is that $I$ takes a minimum value at $V_{b} \approx 1.48 \mathrm{~V}$. This way, largely by the alignment or misalignment of the $\mathrm{N}$ bands of the leads, NDR occurs in the doped nanotubes as shown by the $I-V$ curve of Fig. 2(c). Finally, when $V_{b}$ is increased to $\sim 1.63 \mathrm{~V}$, the contribution of these other bands becomes larger and current increases after the NDR.

The above results of periodic doping are somewhat altered by disorder. As a simplest model, we investigated the effect by shifting position of one of the $\mathrm{N}$ impurities to a new position that is $51.43^{\circ}$ along the nanotube circumference from the periodic $\mathrm{N}$-impurity line. Such a disorder lowers the symmetry of periodically doped tubes. As shown in Fig. 2(a) (solid line), the disorder introduces backscattering that re- duces equilibrium transmission coefficient $T\left(E, V_{b}=0\right)$ as compared with that of periodic doping. In nonequilibrium situation (under a finite bias), shown in Fig. 2(c), for $V_{b}$ $<1.3 \mathrm{~V}$ the disorder reduces current where the main conduction comes from the $\mathrm{N}$ band that is metallic. This reduction of current is consistent with the reduction of transmission coefficient $T\left(E, V_{b}=1.14\right)$ of Fig. 2(d) (solid line). On the other hand, for $V_{b}>1.3 \mathrm{~V}$, the disorder slightly increases the current, similar to that happens in semiconducting nanotubes, where the main conduction comes from the other bands. Indeed, as shown in Fig. $2(\mathrm{~g}), T\left(E, V_{b}=1.63\right)$ (solid line) is reduced for $\mathrm{N}$ band but increased for the other bands.

As discussed above, we expect the transport results obtained from $(7,0)$ nanotubes to hold for other small radii zigzag tubes, and we confirmed this expectation by explicit calculations on doped $(8,0)$ nanotubes. Furthermore, what is the effect of nitrogen doping on metallic nanotubes? Figure $3(\mathrm{c})$ shows a linear $I-V$ curve for $(10,10)$ armchair nanotubes with or without doping, as expected for metallic tubes. ${ }^{12}$ The equilibrium transmission $T\left(E, V_{b}=0\right)$ is shown in Fig. 3(a) together with the band structure [Fig. 3(b)], and they are consistent with known results. ${ }^{4}$ In particular, tubes with one substitutional nitrogen have two dips in $T\left(E, V_{b}=0\right)$, which are due to resonant backscattering by quasibound defect states as investigated before. ${ }^{4}$ Figures $3(\mathrm{~d})$ and $3(\mathrm{~g})$ show nonequilibrium $T\left(E, V_{b}\right)$ in the range of $0<E<V_{b}$ for $V_{b}$ $=0.76 \mathrm{~V}$ and $1.09 \mathrm{~V}$, respectively, for pristine tubes (dotted line) and with single $\mathrm{N}$ impurity (solid line). Up to $V_{b}$ $=0.76 \mathrm{~V}$, there are only $\pi, \pi^{*}$ bands inside the window $\mu_{\max }-\mu_{\min }$ [see Eq. (1) and Figs. 3(e), 3(f)] which contribute to current. The total nonequilibrium transmission $T\left(E, V_{b}=0.76\right)$, Fig. 3(d), for the pure tube is therefore 2, and it is less than 2 for the $\mathrm{N}$ doped tubes due to backscattering. The symbols give transmission coefficient of $\pi^{*}$ and $\pi$ band separately for the doped tube and they are reduced from 


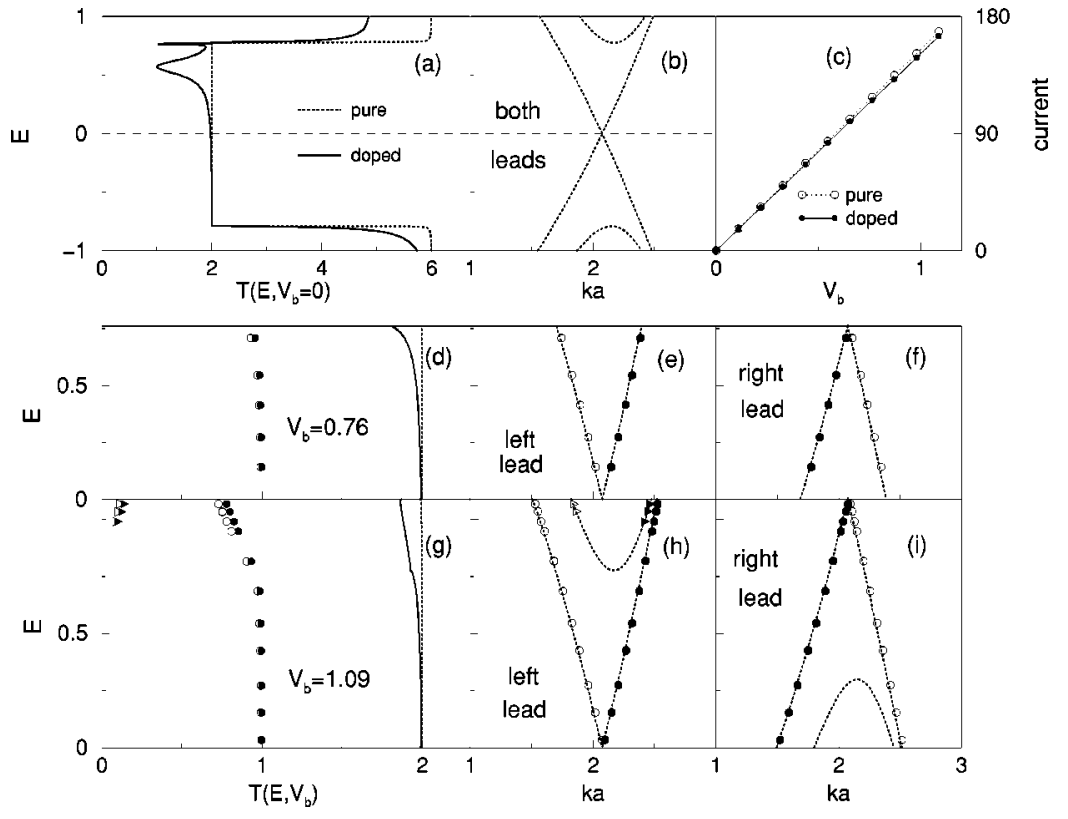

FIG. 3. For pure and single N-doped metallic $(10,10)$ tubes. (a) The equilibrium conductance; (b) the band structure of the lead; (c) the $I-V$ curves; (d), (g) the evolution of the total transmission coefficient $T\left(E, V_{b}\right)$; and (e), (h), (f), (i) for band structures of the left and right leads. Units are the same as in Fig. 1. unity away from $E=0$. The backscattering reduces the current, as shown by the $I-V$ curve [Fig. 3(c)]. When $V_{b}$ $>0.76 \mathrm{~V}$, higher nanotube bands of the left lead start to align with that of the right lead. For a perfect nanotube these higher bands cannot conduct with $\pi^{*}$ and $\pi$ bands due to different rotational symmetry. ${ }^{2,5}$ They do contribute to current for doped tubes because the rotational symmetry is broken by the impurity. For example, at $V_{b}=1.09 \mathrm{~V}$ the triangles in Fig. 3(g) indicate transmission between the higher band of the left lead [indicated by triangles in Fig. 3(h)] and the $\pi^{*}(\pi)$ band of the right lead [Fig. 3(i)]. However, while the current reducing due to a $\mathrm{N}$ doping in $(10,10)$ tubes is small ( $\sim 2 \%$ at $V_{b}=1.09 \mathrm{~V}$ ), larger effect is found for $(4,4)$ tubes $\left(\sim 9 \%\right.$ at $\left.V_{b}=1.09 \mathrm{~V}\right){ }^{22}$ This is reasonable since in the $(4,4)$ tube, the impurity density is higher providing more scattering.

To summarize, we have investigated nonequilibrium transport properties of nitrogen doped carbon nanotubes using $a b$ initio techniques in the model of substitutional doping. The doped zigzag nanotubes show interesting, and somewhat surprising, result that even a single atom substitution has increased the current flow and, for small radii tubes, narrowed the current gap. The physical reason is the break- ing of rotational symmetry by the impurity which allows current to be carried from the hybridization band to the $\pi$ band of the two nanotube leads. Periodical substitution makes zigzag semiconducting tubes metallic, while the band alignment and dispersion effects induce a NDR in the nonlinear $I-V$ curve. For an armchair metallic nanotube, doping with a single impurity reduces current due to elastic backscattering. These results show that breaking the symmetry by impurities can raise the current flow for semiconducting tubes and reduce current for metallic tubes. In this regard, we note that symmetry breaking can be achieved by different means, in addition to impurity doping, other effects including structural imperfection, surrounding one nanotube by other tubes, ${ }^{23}$ or applying a gate voltage, can lower the symmetry of a nanotube device thereby influencing its charge transport properties.

We gratefully acknowledge financial support from the Natural Science and Engineering Research Council of Canada and le Fonds pour la Formation de Chercheurs et l'Aide à la Recherche de la Province du Québec. C.C.K. would like to thank A. McFee McGill Foundation for financial support.
${ }^{1}$ M.S. Dresselhaus, G. Dresselhaus, and P.C. Eklund, Science of Fullerenes and Carbon Nanotubes (Academic, San Diego, 1996); J. Bernholc, C. Roland, and B.I. Yakobson, Crit. Rev. Solid State Mater. Sci. 2, 706 (1997); C. Dekker, Phys. Today 52(5), 22 (1999).

${ }^{2}$ R. Saito, G. Dresselhaus, and M.S. Dresselhaus, Physical Properties of Carbon Nanotubes, (World Scientific, Singapore, 1998).

${ }^{3}$ J.Y. Yi and J. Bernholc, Phys. Rev. B 47, 1708 (1993).

${ }^{4}$ H.J. Choi, J. Ihm, S.G. Louie, and M.L. Cohen, Phys. Rev. Lett.
84, 2917 (2000).

${ }^{5}$ A.A. Farajian, K. Esfarjani, and Y. Kawazoe, Phys. Rev. Lett. 82, 5084 (1999).

${ }^{6}$ F. Leonard and J. Tersoff, Phys. Rev. Lett. 85, 4767 (2000).

${ }^{7}$ Y. Miyamoto, A. Rubio, X. Blase, M.L. Cohen, and S.G. Louie, Phys. Rev. Lett. 74, 2993 (1995).

${ }^{8}$ P.E. Lammert, V.H. Crespi, and A. Rubio, Phys. Rev. Lett. 87, 136402 (2001).

${ }^{9}$ S. Jhi, S.G. Louie, and M.L. Cohen, Phys. Rev. Lett. 85, 1710 (2000); K. Esfarjani, A.A. Farajian, Y. Hashi, and Y. Kawazoe, 
Appl. Phys. Lett. 74, 79 (1999); A. Rubio, Y. Miyamoto, X. Blase, M.L. Cohen, and S.G. Louie, Phys. Rev. B 53, 4023 (1996).

${ }^{10}$ P.L. McEuen, M. Bockrath, D.H. Cobden, Y.G. Yoon, and S.G. Louie, Phys. Rev. Lett. 83, 5098 (1999).

${ }^{11}$ C. Zhou, J. Kong, E. Yenilmez, and H. Dai, Science 290, 1552 (2000); M. Bockrath, W. Liang, D. Bozavic, J.H. Hafner, C.M. Lieber, M. Tinkham, and H. Park, ibid. 291, 283 (2001); M. Bockrath, J. Hone, A. Zettl, P.L. McEuen, A.G. Rinzler, and R.E. Smalley, Phys. Rev. B 61, R10606 (2000); R.S. Lee, H.J. Kim, J.E. Fischer, J. Lefebvre, M. Radosavljevic, J. Hone, and A.T. Johnson, ibid. 61, 4526 (2000); R.D. Antonov and A.T. Johnson, Phys. Rev. Lett. 83, 3274 (1999); P.G. Collins, K. Bradley, M. Ishigami, and A. Zettl, Science 287, 1801 (2000); Y. Cui, Q. Wei, H. Park, and C.M. Lieber, ibid. 293, 1289 (2001).

${ }^{12}$ J. Taylor, H. Guo, and J. Wang, Phys. Rev. B 63, 121104 (2001); 63, 245407 (2001).

${ }^{13}$ In the numerics, the device scattering region consists of three unit cells (12 carbon rings) for a total of 84 atoms for the $(7,0)$ nanotube, and 2.5 unit cells (five carbon rings) of a total of 100 atoms for the $(10,10)$ nanotube. On the other hand, the nanotube electrodes where the bias is applied and current collected extend to $z= \pm \infty$. Our DFT uses $s, p, d$ atomic orbital basis [see, for example, P. Ordejón, E. Artacho, and J.M. Soler, Phys. Rev. B 53, 10441 (1996)]; local density approximation, and standard psuedopotentials [G.B. Bachelet, D.R. Hamann, and M. Schlüter, ibid. 26, 4199 (1982)]. Due to a finite bias voltage $V_{b}$, we construct charge density using NEGF with $V_{b}$ providing the boundary condition of the Poisson equation which we solve in real space. For technical details, see Ref. 12.

${ }^{14}$ A.P. Jauho, N.S. Wingreen, and Y. Meir, Phys. Rev. B 50, 5528 (1994).

${ }^{15}$ S. Datta, Electronic Transport in Mesoscopic Systems (Cambridge University Press, New York, 1995).

${ }^{16}$ B.G. Wang, J. Wang, and H. Guo, Phys. Rev. Lett. 82, 398 (1999); J. Appl. Phys. 86, 5094 (1999).

${ }^{17}$ Our band structures are consistent with Ref. 7. See also, H.J. Choi, and J. Ihm, Phys. Rev. B 59, 2267 (1999).

${ }^{18}$ X. Blase, L.X. Benedict, E.L. Shirley, and S.G. Louie, Phys. Rev. Lett. 72, 1878 (1994).

${ }^{19}$ L. Chico, L.X. Benedict, S.G. Louie, and M.L. Cohen, Phys. Rev. B 54, 2600 (1996).

${ }^{20}$ There is one band in Figs. 1(h) and 1(i), which has tiny conduction even for doped tubes. This should be attributed to its small group velocity.

${ }^{21}$ Fisher-Lee relation gives the transmission coefficient $T$ in terms of the Green's function $G^{R}, T \sim v_{l} v_{r}\left|G^{R}\right|^{2}$. See, D.S. Fisher and P.A. Lee, Phys. Rev. B 23, 6851 (1981).

${ }^{22}$ For $(4,4)$ nanotube device, we have numerically checked that scattering regions containing five carbon rings (40 atoms) and nine carbon rings (72 atoms) give essentially the same transport results.

${ }^{23}$ P. Delaney, H.J. Choi, J. Ihm, S.G. Louie, and M.L. Cohen, Nature (London) 391, 466 (1998). 